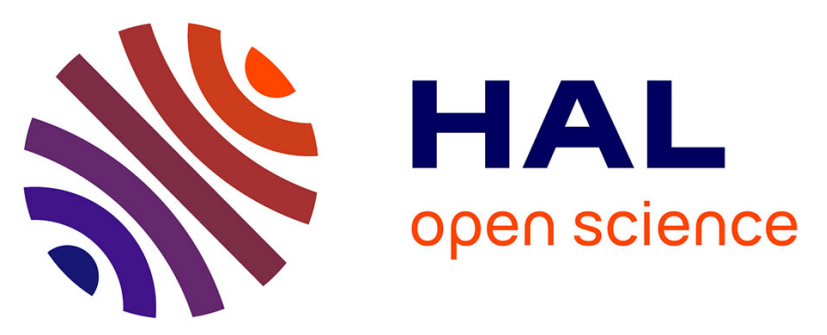

\title{
ÉTUDE DES PICS DE FROTTEMENT INTÉRIEUR OBSERVÉS APRÈS ÉCROUISSAGE SUR LE TITANE ET LE ZIRCONIUM, DANS LE DOMAINE DES BASSES TEMPÉRATURES
}

\author{
J. Petit, Michel Quintard, R. Soulet, J. de Fouquet
}

\section{To cite this version:}

J. Petit, Michel Quintard, R. Soulet, J. de Fouquet. ÉTUDE DES PICS DE FROTTEMENT INTÉRIEUR OBSERVÉS APRÈS ÉCROUISSAGE SUR LE TITANE ET LE ZIRCONIUM, DANS LE DOMAINE DES BASSES TEMPÉRATURES. Journal de Physique Colloques, 1971, 32 (C2), pp.C2-215-C2-220. 10.1051/jphyscol:1971247 . jpa-00214573

HAL Id: jpa-00214573

https://hal.science/jpa-00214573

Submitted on 1 Jan 1971

HAL is a multi-disciplinary open access archive for the deposit and dissemination of scientific research documents, whether they are published or not. The documents may come from teaching and research institutions in France or abroad, or from public or private research centers.
L'archive ouverte pluridisciplinaire HAL, est destinée au dépôt et à la diffusion de documents scientifiques de niveau recherche, publiés ou non, émanant des établissements d'enseignement et de recherche français ou étrangers, des laboratoires publics ou privés. 


\title{
ÉTUDE DES PICS DE FROTTEMENT INTÉRIEUR OBSERVÉS APRẼS ÉCROUISSAGE SUR LE TITANE ET LE ZIRCONIUM, DANS LE DOMAINE DES BASSES TEMPÉRATURES
}

\author{
J. PETIT, M. QUINTARD, R. SOULET et J. de FOUQUET
}

Laboratoire de Mécanique et Physique des Matériaux, E. N. S. M. A., Poitiers

\begin{abstract}
Résumé. - Des mesures de frottement intérieur effectuées en vibrations longitudinales sur du Zirconium et du Titane non alliés à des fréquences de l'ordre de $20 \mathrm{kHz}$, ont montré l'existence après écrouissage de pics de frottement intérieur entre $-60^{\circ} \mathrm{C}$ et $+50^{\circ} \mathrm{C}$. Les caractéristiques de ces pics et leur évolution en fonction du taux de déformation, des recuits isochrones, de la pureté du métal et de l'état initial, ont été établies.

Abstract. - Internal friction measurements have been made at about $20 \mathrm{kHz}$ by longitudinal vibrations in Zirconium and Titane. Peaks have been observed after cold-working between $-60^{\circ}$ and $+50^{\circ} \mathrm{C}$. The characteristics of the peaks, and the dependence of the decrement on plastic deformation, isochronal anneals, purity of metals, and initial state, have been established.
\end{abstract}

I. Introduction. - Une étude antérieure faite sur des alliages zirconium-cuivre, nous avait permis de mettre en évidence l'existence d'un pic de frottement intérieur situé vers $320^{\circ} \mathrm{K}$ sur le métal écroui, pour une fréquence de vibration de $13 \mathrm{kHz}$ [1]. Ce pic présentait des caractéristiques permettant de l'assimiler à un pic de Bordoni [2]. Nous avons alors entrepris une étude du zirconium de différentes puretés afin de préciser les conditions d'obtention de ce pic et ses caractéristiques. Une étude parallèle a été faite sur le Titane de façon à comparer dans des conditions identiques les spectres obtenus sur les deux métaux.

II. Appareillage expérimental. - Les mesures de frottement intérieur sont effectuées sur des barreaux cylindriques vibrant longitudinalement à des fréquences allant de 15 à $25 \mathrm{kHz}$, dans une enceinte sous vide $\left(<10^{-5}\right.$ torr) [3]. La température est mesurée à l'aide d'un thermocouple qui touche l'éprouvette au nœud de vibration. Elle peut évoluer de $-190^{\circ}$ à $+200^{\circ} \mathrm{C}$ sous le contrôle d'un programmateur.

Nous avons utilisé deux nuances de chaque métal (tableau I)

III. Zirconium. - ZIRCONIUM 99,9\% (J. M.). Les barreaux utilisés sont des cylindres de diamètre $3 \mathrm{~mm}$ et de longueur $100 \mathrm{~mm}$, vibrant à des fréquences de l'ordre de $2 \times 10^{4} \mathrm{~Hz}$.

Etat «brut », - L'amortissement du métal «brut », a été déterminé afin de vérifier si les éprouvettes étaient identiques. On observe un pic culminant vers $-\underline{45^{\circ} \mathrm{C}}$ pour une valeur $\mathrm{du}$ frottement intérieur

$$
Q_{\max }^{-1}=50 \times 10^{-5}
$$

dont le sommet est légèrement dédoublé (Fig. 1).

\section{TABLEAU I}

Les analyses des métaux fournies par Johnson Matthey ne portent pas sur les inclusions gazeuses. Seules figurent les impuretés de concentrations supérieures au $p . p . m$.

Impuretés Zirconium Zirconium Ti JM Ti MRC $\begin{array}{lllllll}\text { enp.p.m. JM 99,9 \% } & \text { MRC } 99,99 \% & 99,999 \% & 99,97 \%\end{array}$

- - - $\quad$ -

$\begin{array}{ccrrr}\mathrm{C} & ? & 6 & ? & 78 \\ \mathrm{H} & ? & 4 & ? & 3 \\ \mathrm{O} & ? & 63 & ? & 125 \\ \mathrm{~N} & ? & 6 & ? & 2 \\ \mathrm{Al} & 30 & 15 & - & - \\ \mathrm{Cr} & - & 12 & - & - \\ \mathrm{Cu} & 10 & 8 & 5 & - \\ \mathrm{Fe} & 500 & 30 & 3 & 30 \\ \mathrm{Hf} & <200 & 40 & - & 12 \\ \mathrm{Mg} & 5 & - & - & <15 \\ \mathrm{Mn} & 2 & - & - & - \\ \mathrm{Mo} & - & - & - & <5 \\ \mathrm{Nb} & - & - & - & 2 \\ \mathrm{Ni} & - & 1,5 & - & 2 \\ \mathrm{Si} & 40 & 1,5 & -2 & - \\ \mathrm{Ti} & 20 & 1 & - & - \\ \mathrm{Sn} & 20 & - & - & <10 \\ \text { Autres } & & <10 & & <10\end{array}$

Etat recuit. - Afin de suivre l'évolution du pic nous avons effectué un premier maintien d'une heure à $250^{\circ} \mathrm{C}$; le pic s'élève et se déplace vers les hautes températures : $Q_{\max }^{-1}=59 \times 10^{-5}$ et $T_{\max }^{0}=-38^{\circ} \mathrm{C}$. Après une heure à $400^{\circ} \mathrm{C}, Q_{\max }^{-1}=21 \times 10^{-5}$ et $T_{\max }^{\mathrm{a}}=-43^{\circ} \mathrm{C}$. Un recuit de deux heures à $675^{\circ} \mathrm{C}$ fait disparaître le pic et $Q^{-1}$ est inférieur à $2 \times 10^{-5}$ (Fig. 1).

Influence de l'écrouissage. - La déformation est obtenue par traction à la température ambiante. Les 


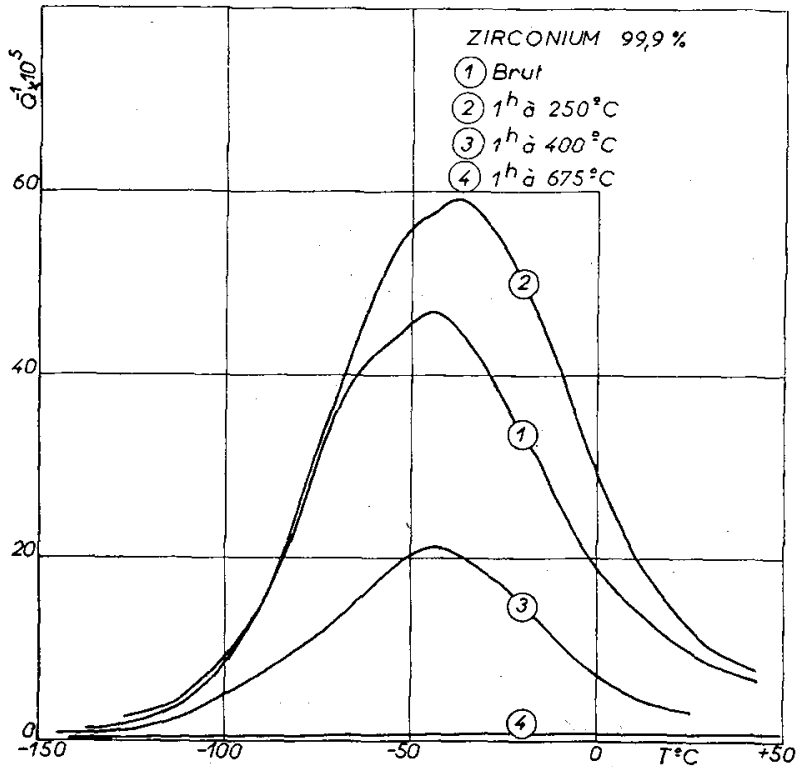

Fig. 1. - Influence des recuits isochrones sur le pic observé sur le Zirconium $99,9 \%$ écroui.

éprouvettes sont mises à froid une demi-heure après écrouissage. L'évolution du pic en fonction du taux de déformation permanente $\varepsilon_{p}$ est représentée figure 2 .

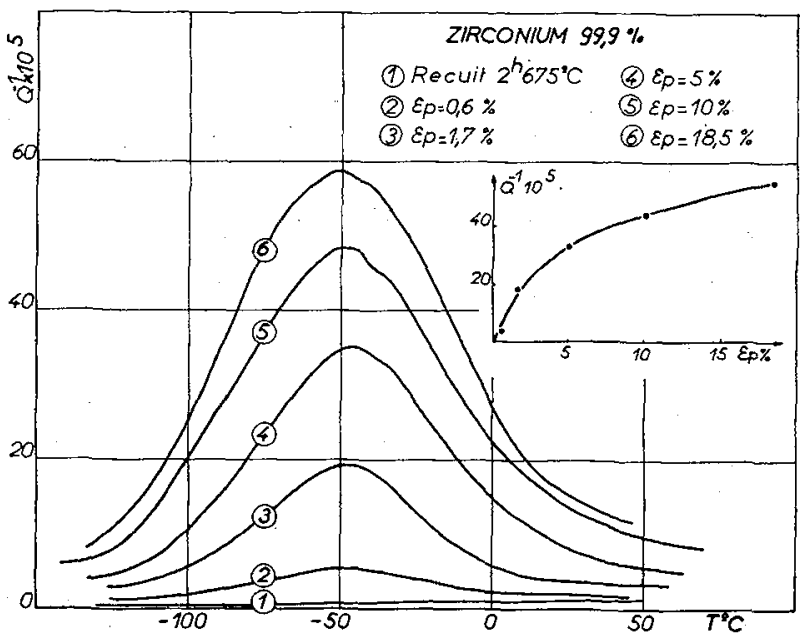

Fig. 2. - Infiuence de l'écrouissage préalable sur le pic observé sur le Zirconium 99,9\%.

La température du sommet du pic est sensiblement la même que sur le métal brut, soit voisine de $-45^{\circ} \mathrm{C}$. On notera, comme sur l'état brut, le dédoublement du pic, et la diminution de la température du sommet du pic quand $\varepsilon_{p}$ augmente [4].

Vieillissement. - La figure 3 représente les variations du pic en fonction des recuits cumulatifs de $1 \mathrm{~h}$, à des températures croissantes, sur une éprouvette déformée de $10 \%$. On constate que $Q_{\max }^{-1}$ augmente jusqu'à $T_{R}=325^{\circ} \mathrm{C}$ avec un minimum vers $250^{\circ} \mathrm{C}$. Comme sur le métal brut, le sommet se déplace jusqu'à $-38^{\circ} \mathrm{C}$, et revient ensuite jusqu'à $-50^{\circ} \mathrm{C}$ pour

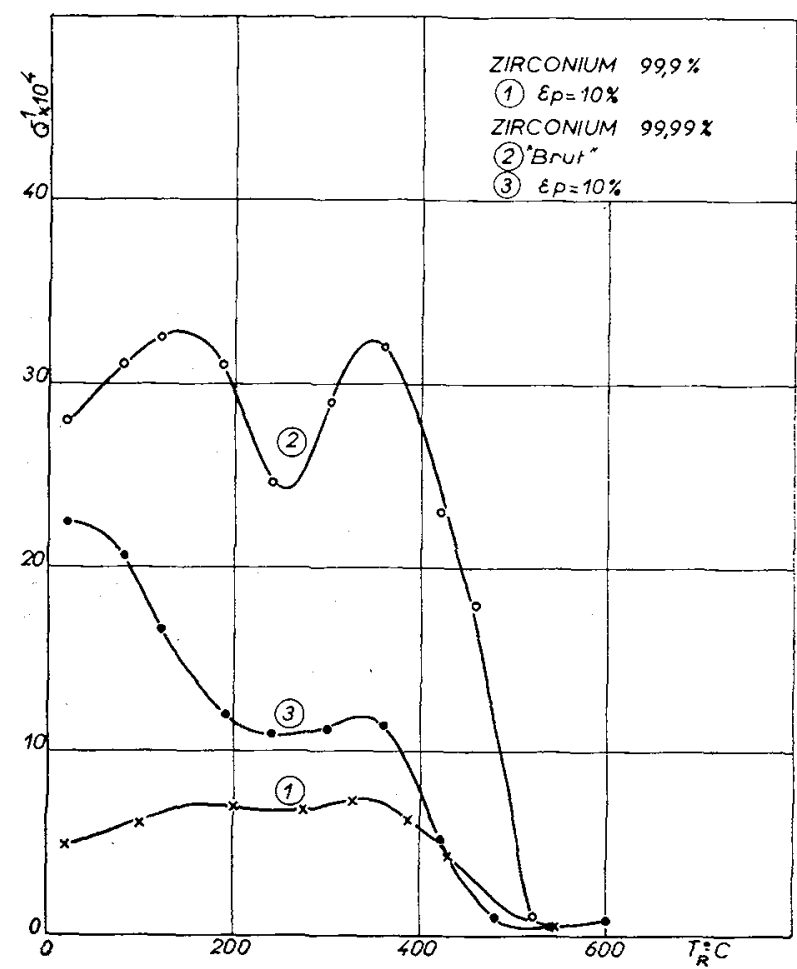

FIG. 3. - Intensité maximum de relaxation en fonction de la température de recuit : Zirconium 99,9 et $99,99 \%$.

$T_{R}=525^{\circ} \mathrm{C}$ alors qu'il ne reste plus qu'un léger maximum. On peut également noter qu'après une heure à $425^{\circ} \mathrm{C}$ le pic est de même hauteur que sur le métal écroui de $10 \%$, mais moins large, et culmine à une température sensiblement plus élevée.

ZIRCONIUM 99,99 \% (MRC). — Les éprouvettes sont de même longueur que précédemment $(100 \mathrm{~mm})$ (elles vibrent donc à des fréquences de même ordre) mais ont un diamètre de $6,5 \mathrm{~mm}$ ce qui a permis d'effectuer les écrouissages inférieurs à $5 \%$ en compression, c'est-à-dire sans perte de métal (en traction, les têtes doivent être enlevées).

Etat «brut». - Le spectre du frottement intérieur obtenu sur six éprouvettes différentes, montre comme pour le métal $3 \mathrm{~N}$ un pic culminant vers $-45^{\circ} \mathrm{C}$ et de sommet légèrement dédoublé, mais d'amplitude très supérieure $\left(28\right.$ à $\left.40 \times 10^{-4}\right)$ soit 6 à 8 fois plus élevée.

Recuit du métal «brut». - Des recuits isochrones de trois heures à température $T_{R}$ croissante donnent une évolution similaire à celle du métal $3 \mathrm{~N}$ (Fig. 3). Nous observons tout d'abord une élévation du pic, associée à un déplacement du sommet de quelques degrés vers les hautes températures (jusqu'à $\left.T_{R}=120^{\circ} \mathrm{C}\right) . \mathrm{La}$ décroissance observée au-delà, accompagnée d'un abaissement du "fond continu », est suivie à partir de $T_{R}=240^{\circ} \mathrm{C}$ d'une réaugmentation du pic. Puis, au-delà de $T_{R}=360^{\circ} \mathrm{C}$, le pic décroît rapidement. 
Influence de l'écrouissage. - L'évolution du pic en fonction du taux $\varepsilon_{p}$ de déformation permanente, sur le métal recuit $3 \mathrm{~h}$ à $800^{\circ} \mathrm{C}$ sous ultra-vide, est représentée figure 4 . On constate que si pour le métal

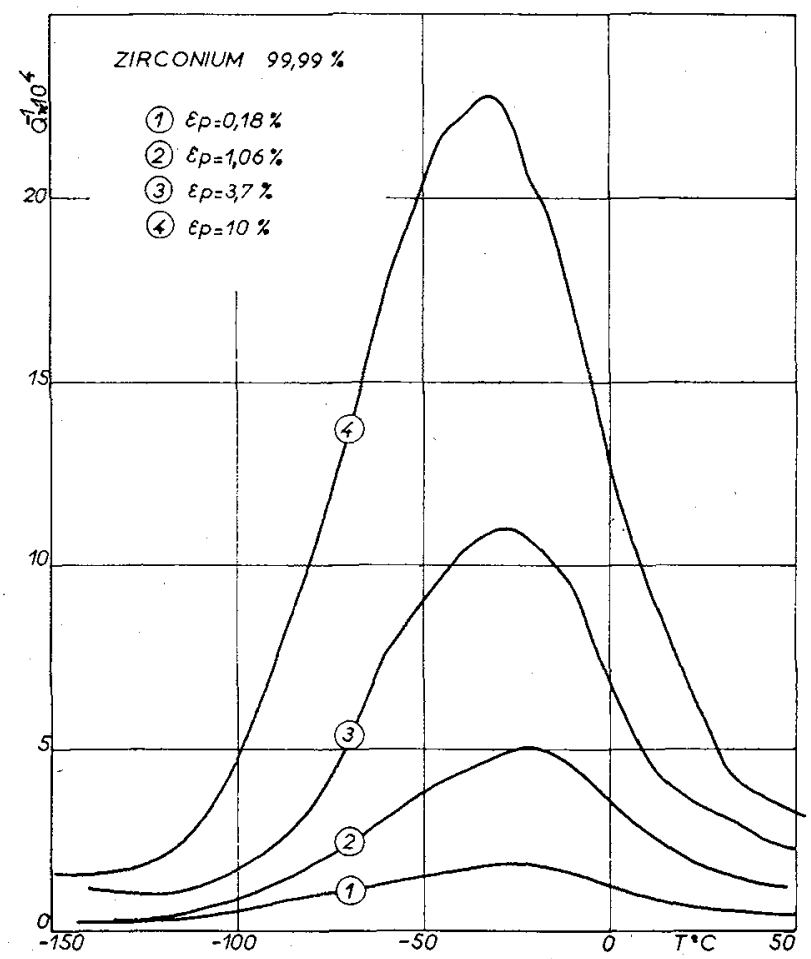

Fig. 4. - Influence de l'écrouissage préalable sur le pic observé sur le Zirconium 99,99\%.

brut les pics étaient à la même température pour les deux puretés $\left(\simeq-45^{\circ} \mathrm{C}\right)$, ici le maximum du pic est situé vers $-30^{\circ} \mathrm{C}$. De plus, à écrouissage égal, l'amplitude est cinq fois plus élevée pour le zirconium le plus pur; la température du sommet décroît lorsque $\varepsilon_{p}$ augmente.

Des essais systématiques ont montré que la position du pic était sensible aux recuits avant déformation ; en particulier des recuits cumulatifs prolongés (tels ceux mentionnés plus haut : III-2-b) et des recuits sous vides moléculaires conduisent à des températures et des hauteurs de pic intermédiaires, cet effet étant très probablement dû à une contamination du métal.

Vieillissement. - Des recuits isochrones de $3 \mathrm{~h}$ à $T_{R}$ sur un échantillon déformé de $10 \%$ montrent (Fig. 3) une évolution du pic qui diffère sensiblement de celle observée à l'état «brut » et sur le métal de pureté $3 \mathrm{~N}$. Le frottement intérieur diminue dès les premiers maintiens, jusqu'à $T_{R}=240^{\circ} \mathrm{C}$, tandis que la température du sommet évolue de $-32{ }^{\circ} \mathrm{C}$ à $-40^{\circ} \mathrm{C}$. Le minimum à $240^{\circ} \mathrm{C}$ est suivi d'une légère élévation du pic jusqu'à $T_{\mathrm{R}}=360^{\circ} \mathrm{C}$, le sommet demeurant à $-40^{\circ} \mathrm{C}$. Puis le pic s'estompe, son sommet évoluant vers $-45^{\circ} \mathrm{C}$.

Discussion. - L'ensemble des mesures précédentes montre l'existence dans tous les cas d'un spectre de frottement intérieur dû à l'écrouissage, qui s'étend de $-130^{\circ} \mathrm{C}$ à $+50^{\circ} \mathrm{C}$, mais beaucoup mieux défini que celui obtenu sur le magnésium par Tsui et Sack [7]. Cependant la position du pic et son amplitude dépendent considérablement de la pureté, en particulier pour les métaux très purs. La figure 5 montre à titre de

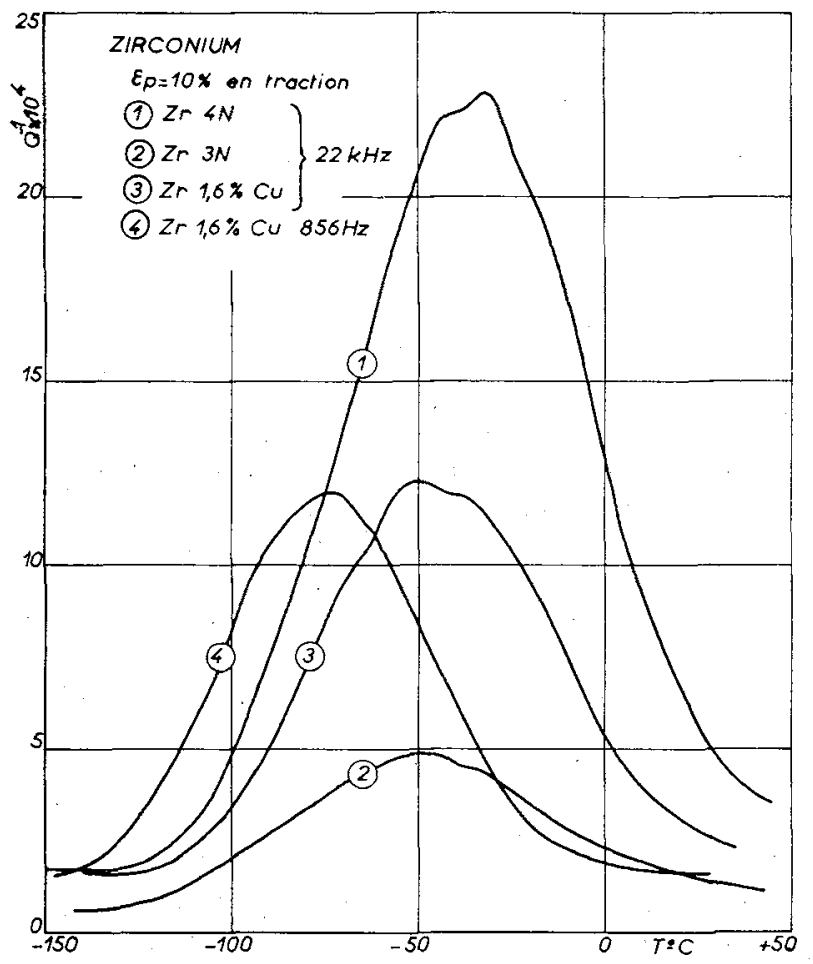

Fig. 5. - Différents pics obtenus sur le $\mathrm{Zr} 99,9$ et $99,99 \%$ et sur un alliage $\mathrm{Zr}-\mathrm{Cu}$ à $1,6 \%$ de $\mathrm{Cu}$ écrouis de $10 \%$.

comparaison, les courbes obtenues sur $\mathrm{Zr} 4 \mathrm{~N}, \mathrm{Zr}$ $3 \mathrm{~N}$ et $\mathrm{Zr}-\mathrm{Cu} 1,6 \%$ déformés de $10 \%$ après recuit. On remarque que le pic $\mathrm{Zr} 4 \mathrm{~N}$ est décalé d'environ $15^{\circ} \mathrm{C}$ vers les hautes températures par rapport aux deux autres qui sont centrés vers $-45^{\circ} \mathrm{C}$; d'autre part le pic $\mathrm{Zr} 3 \mathrm{~N}$ est beaucoup plus faible que les deux autres. L'étude faite antérieurement sur les alliages $\mathrm{Zr}-\mathrm{Cu}$ [1] montrait une évolution de $Q^{-1}$ en fonction de $\varepsilon_{p}$ très analogue à celle observée sur $\mathrm{Zr} 3 \mathrm{~N}$, le rapport d'amplitude, de l'ordre de 2,5, restant constant au cours de l'écrouissage.

Nous avons évalué pour les deux nuances la valeur moyenne de l'énergie d'activation $\mathrm{H}_{\mathrm{eV}}$.

Pour $\mathrm{Zr} 3 \mathrm{~N}$ nous nous sommes référés à une étude de Hasiguti [5] qui a obtenu sur un métal de même pureté, écroui par laminage, un pic situé à $205^{\circ} \mathrm{K}$ pour une fréquence de sollicitation de $1 \mathrm{kHz}$; à l'aide de nos résultats, nous obtenons

$$
H_{\mathrm{eV}}=0,50 \pm 0,05 \mathrm{eV} \text {. }
$$

Pour l'alliage $\mathrm{Zr}-\mathrm{Cu} 1,6 \%$ nous avons effectué des mesures en "flexion", à une fréquence de $856 \mathrm{~Hz}$, sur un barreau issu du même matériau que celui utilisé en «traction compression» et déformé de 
façon identique de $10 \%$; nous avons obtenu un pic culminant à $1990^{\circ} \mathrm{K}$ (valeur proche de celle donnée par Hasiguti). La valeur moyenne de l'énergie d'activation est alors $H_{\mathrm{ev}}=0,47 \pm 0,05 \mathrm{eV}$. Ces deux valeurs sont très voisines, mais par contre diffèrent notablement de la valeur annoncée par Hasiguti, soit $0,18 \pm 0,02 \mathrm{eV}$.

D'autre part F. Povolo et E. Bisogni [6] ayant déformé de $10 \%$ une éprouvette de zirconium $4 \mathrm{~N}$ après un recuit de $2 \mathrm{~h}$ à $750^{\circ} \mathrm{C}$ (sous vide $10^{-6}$ torr) signalent la présence d'un pic à $144^{\circ} \mathrm{K}$

$$
\left(Q_{\max }^{-1}=30 \times 10^{-4}\right)
$$

pour une fréquence de $1 \mathrm{~Hz}$. Nous obtenons, dans des conditions très proches, un pic à $-32{ }^{\circ} \mathrm{C}$ sur $\mathrm{Zr}$ 4N. L'énergie d'activation est alors évaluée à $0,30 \pm 0,03 \mathrm{eV}$.

Ces résultats suggèrent l'existence d'un pic vers $-45^{\circ} \mathrm{C}$, que l'on observe seul sur $\mathrm{Zr} 3 \mathrm{~N}$ et $\mathrm{Zr}-\mathrm{Cu}$, et d'un second pic, situé à plus haute température, qui devient prépondérant pour le métal le plus pür soit $\mathrm{Zr} 4 \mathrm{~N}$. L'existence du seul pic à $-45^{\circ} \mathrm{C}$ sur le $\mathrm{Zr} 4 \mathrm{~N}$ « brut » résulterait d'un état fortement écroui dans des conditions qui nous sont inconnues, probablement à une température nettement supérieure à l'ambiante.

IV. Titane. - Etat «brut». - Le spectre obtenu sur le titane $99,97 \%$ 《brut » est analogue à celui observé sur les zirconiums «bruts »; il est cependant un peu plus élevé et situé à une température plus basse ( $-50^{\circ} \mathrm{C}$ à $16,5 \mathrm{kHz}$ ) (Fig. 6). Par contre, pour

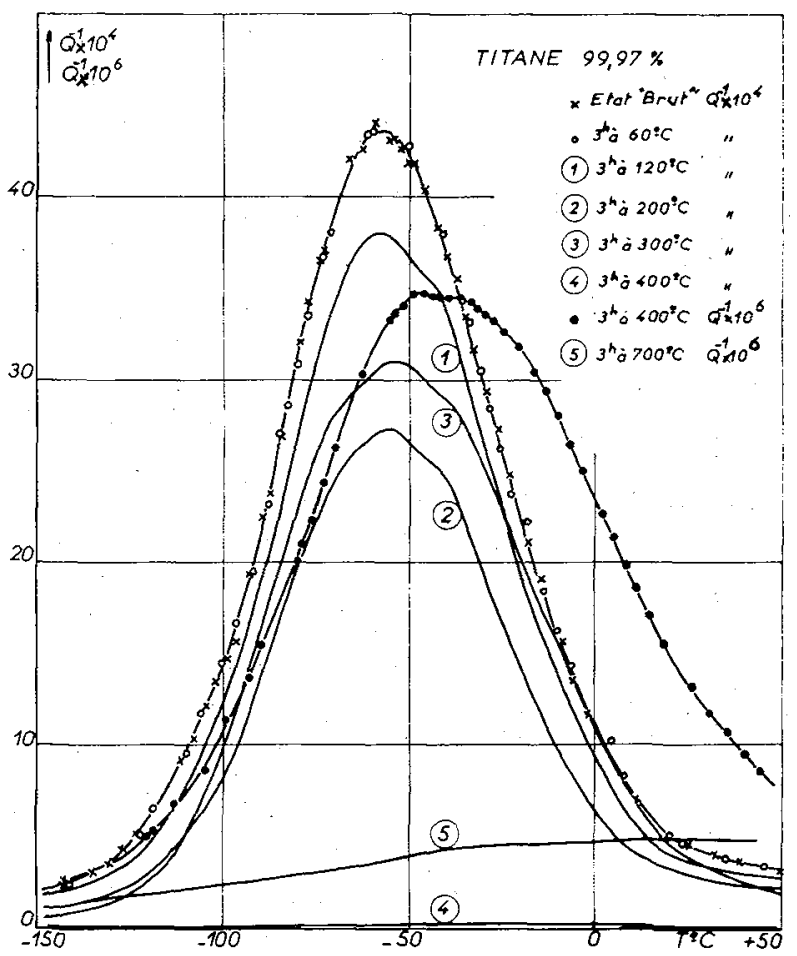

FIG. 6. - Influence des recuits isochrones sur le pic observé sur le Titane $99,9 \%$ écroui. le métal $5 \mathrm{~N}$, on observe un pic de $0^{\circ} \mathrm{C}$ avec toutefois un maximum relatif vers $-60^{\circ} \mathrm{C}$ (Fig. 7).

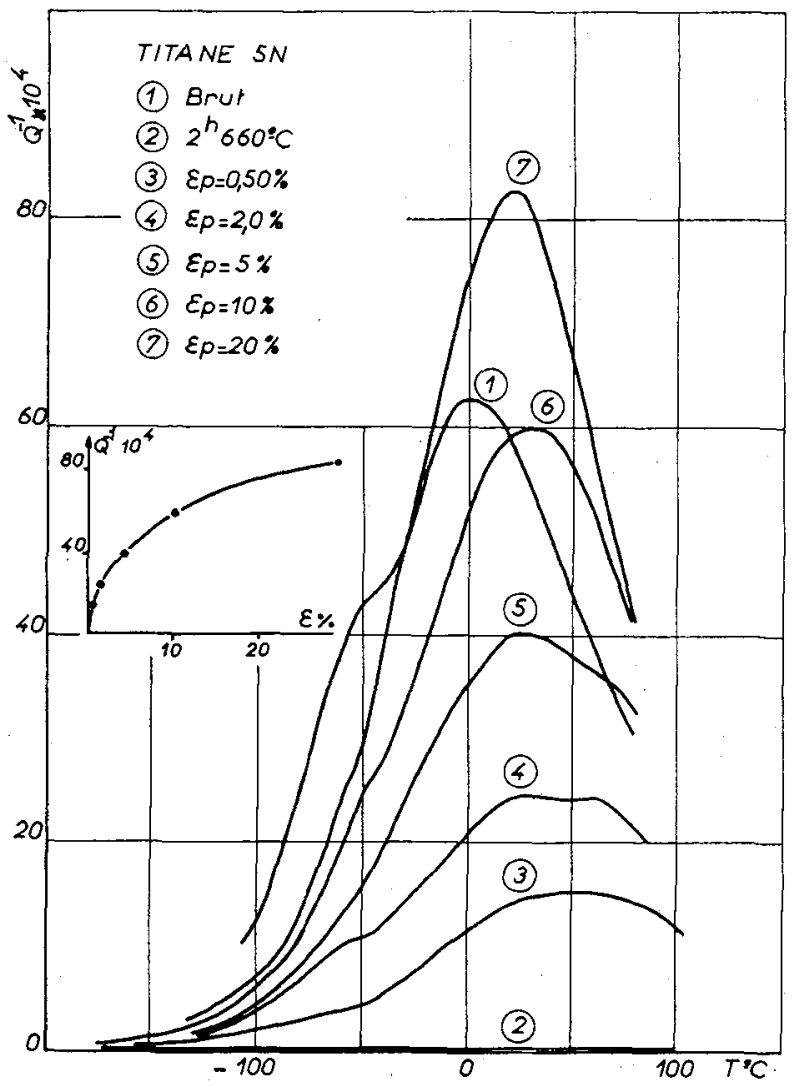

Fig. 7. - Influence de l'écrouissage sur le pic observé sur le Titane $99,999 \%$.

La figure 6 montre l'évolution de la hauteur du pic du titane $99,97 \%$ en fonction des recuits isochrones. Nous noterons d'une part la remontée du pic après $3 \mathrm{~h}$ à $300^{\circ} \mathrm{C}$ et d'autre part la courbe obtenue après $3 \mathrm{~h}$ à $400^{\circ} \mathrm{C}$ où le frottement intérieur est 100 fois plus faible qu'à l'état initial.

Influence de l'écrouissage. - Les variations du pic en fonction du taux de déformation son représentée sur la figure 7 pour $\mathrm{Ti} 5 \mathrm{~N}$ et sur la figure 8 pour $\mathrm{Ti}$ $99,97 \%$. Ces courbes appellent différentes remarques :

$1^{\circ}$ l'analogie des spectres avec ceux obtenus sur le zirconium $4 \mathrm{~N}$.

$2^{\circ}$ l'existence d'un maximum relatif dans tous les cas vers $-60^{\circ} \mathrm{C}$;

$3^{\circ}$ la présence d'un ou plusieurs pics situés à des températures plus élevées (entre $-25^{\circ} \mathrm{C}$ et $+50^{\circ} \mathrm{C}$ selon la pureté et le taux de déformation) et dont la température du maximum de l'ensemble se déplace vers les basses températures quand l'écrouissage augmente.

Vieillissement. - L'évolution du maximum du frottement intérieur au cours du recuit est indiquée figure 9. 


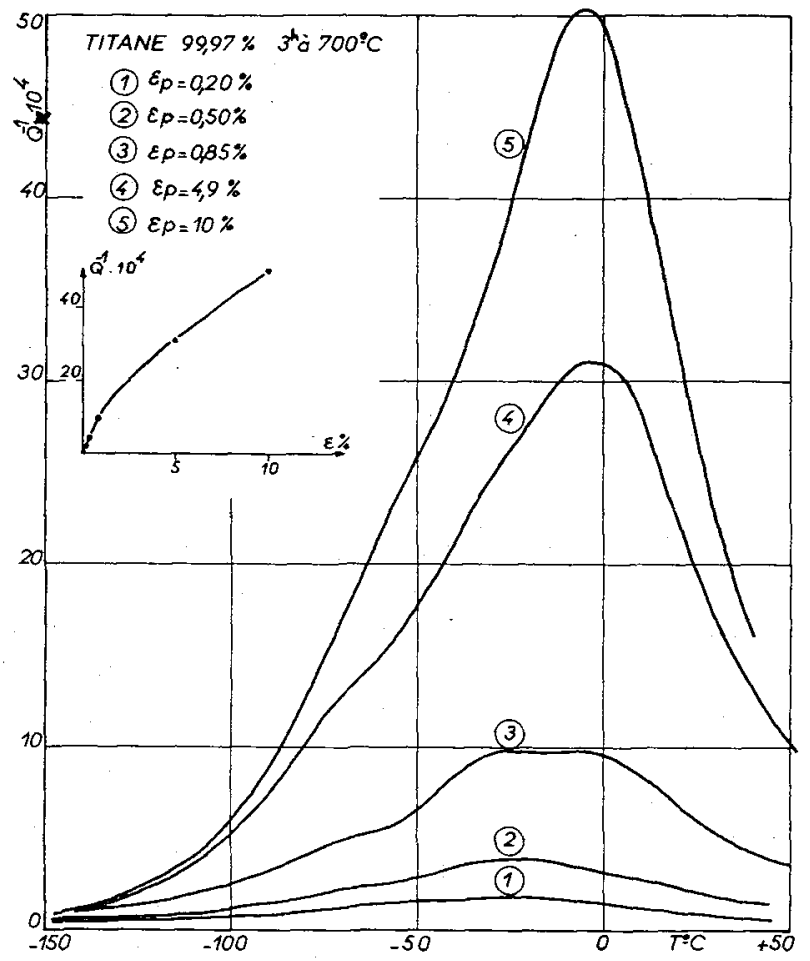

Fig. 8. - Influence de l'écrouissage sur le pic observé sur le Titane $99,9 \%$.

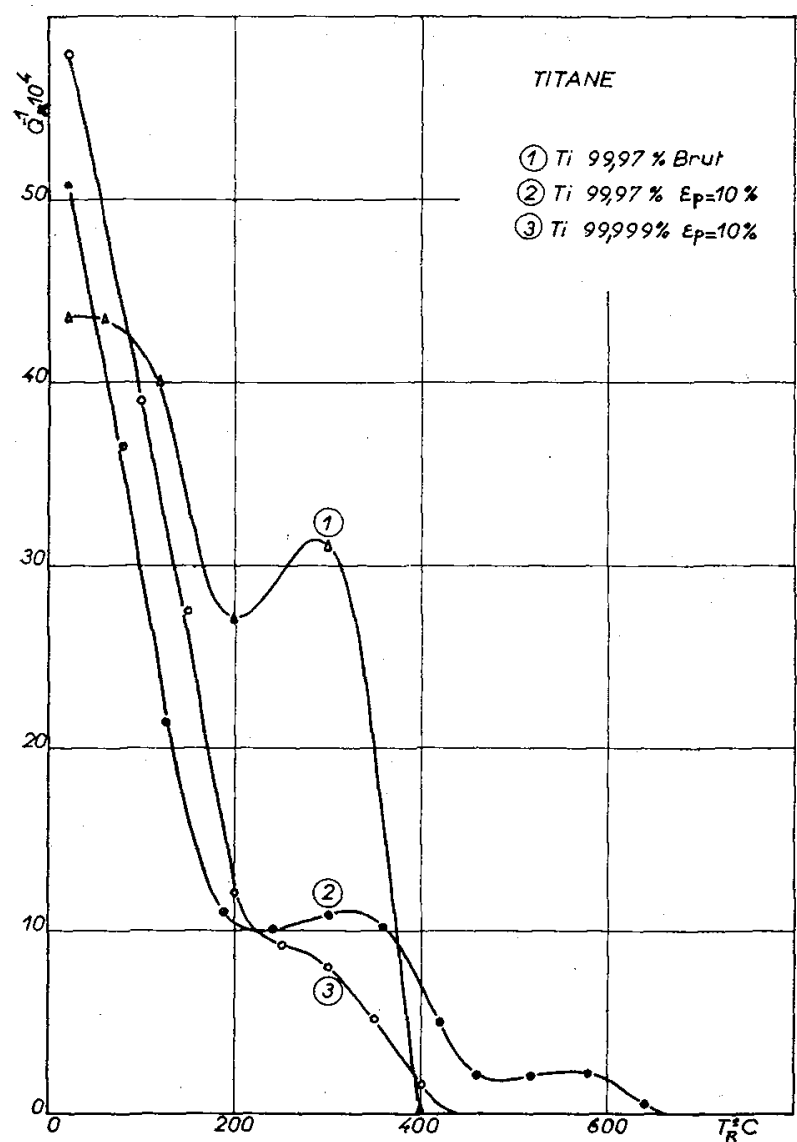

FIG. 9. - Intensité maximum de relaxation en fonction de la température de recuit : Titane $99,9 \%$ et $99,999 \%$.
Dans tous les cas on observe une décroissance rapide (un peu moins pour le métal "brut») jusque vers $200^{\circ} \mathrm{C}$, suivie d'un palier (et même d'une remontée pour le «brut») puis d'une nouvelle décroissance jusqu'à $450^{\circ} \mathrm{C}$ et d'un second palier pour Tí 99,97 écroui de $10 \%$. Les mesures effectuées sur Ti $5 \mathrm{~N}$ audelà de $450^{\circ} \mathrm{C}$ ayant été effectuées sur des barreaux de faible diamètre, c'est-à-dire dans des conditions difficiles, il est possible que le second palier n'ait pas pu être décelé.

Les figures 10 et 11 montrent l'évolution du spectre d'amortissement obtenu sur le titane $99,97 \%$ déformé de $10 \%$. Ces courbes mettent en évidence l'évolution de la température du maximum : $-6^{\circ} \mathrm{C}$ après écrouissage, $-20^{\circ} \mathrm{C}$ après $3 \mathrm{~h}$ à $190^{\circ} \mathrm{C}\left(Q^{-1}\right.$ étant cinq fois plus faible). De $190^{\circ} \mathrm{C}$ à $360^{\circ} \mathrm{C} Q^{-1}$ est pratiquement constant tandis que le sommet évolue de $-20^{\circ} \mathrm{C}$ à $-45^{\circ} \mathrm{C}$. Le second palier correspond à un pic centré vers $-70^{\circ} \mathrm{C}$ avec $Q^{-1}$ de l'ordre de $2 \times 10^{-4}$. A $640^{\circ} \mathrm{C}$ il demeure une bosse vers $-65^{\circ} \mathrm{C}$. Il semble donc qu'il faille atteindre le début de la recristallisation pour que le pic disparaisse complètement.

Discussion. - Les résultats obtenus sur le titane sont donc comparables à ceux relatifs au zirconium. Pour des fréquences de même ordre, l'étendue du spectre de relaxation observé après écrouissage est cependant plus large sur le titane, en particulier pour le métal le plus pur. Dans le cas du zirconium nous avons été amenés à considérer l'existence de deux pics, l'un vers $-45^{\circ} \mathrm{C}$ et l'autre vers $-30^{\circ} \mathrm{C}$. Dans le cas du titane les résultats ci-dessus suggèrent l'exis-

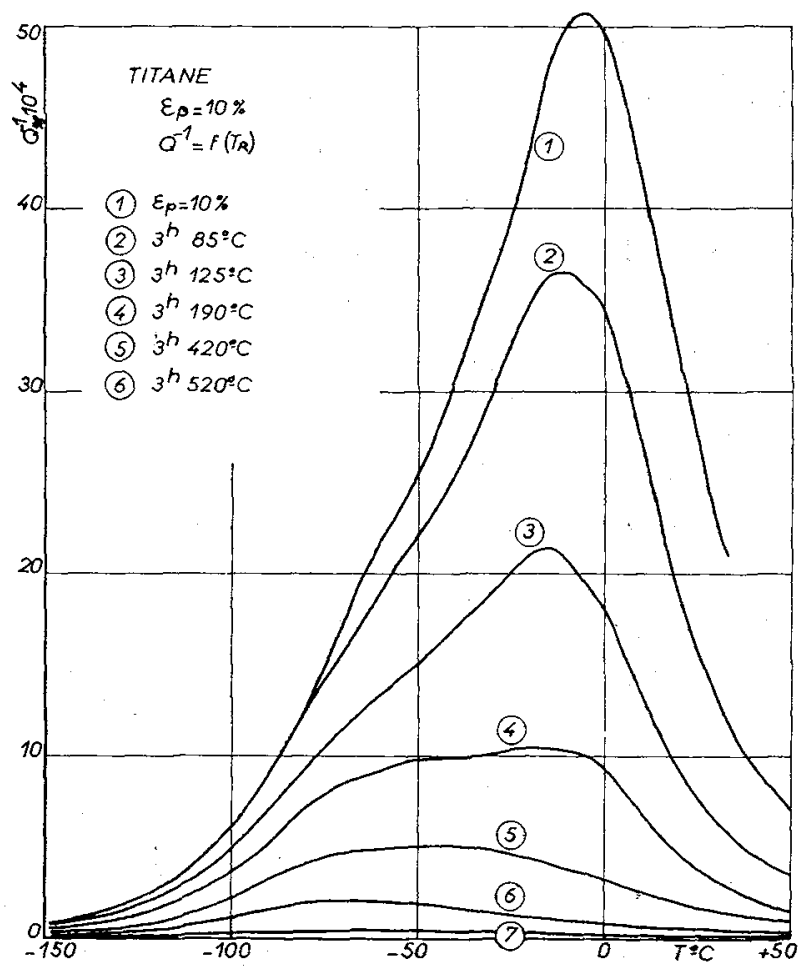

FIG. 10. - Influence des recuits isochrones sur le pic observé sur le Titane $99,9 \%$ [1], après un écrouissage préalable de $10 \%$. 


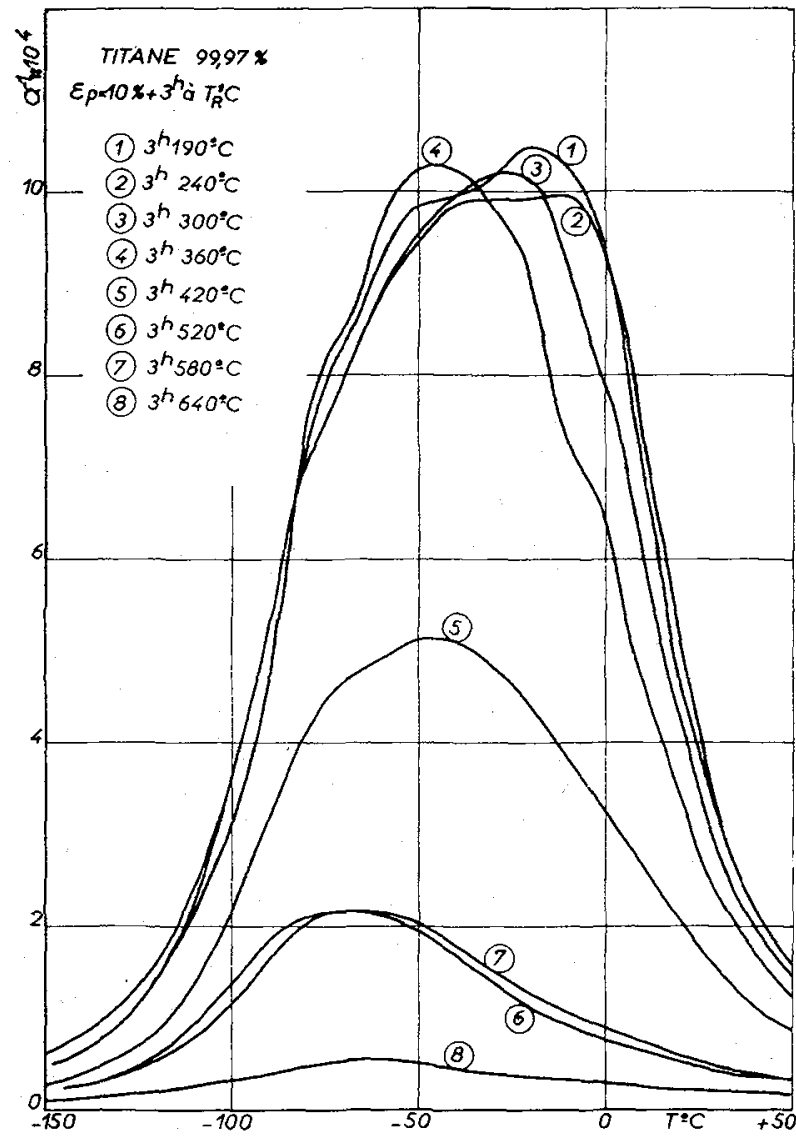

FIG. 11. - Influence des recuits isochrones sur le pic observé sur le Titane 99,9\% [2] après un écrouissage préalable de $10 \%$. tence d'un pic vers $-60^{\circ} \mathrm{C}$, que l'on observe seul sur le Ti $99,97 \%$ «brut» (de structure comparable à celle du $\mathrm{Zr} 4 \mathrm{~N}$ ) auquel viennent se superposer un ou plusieurs pics dont le maximum apparent se déplace de -25 à $+50^{\circ} \mathrm{C}$ selon l'écrouissage et la pureté. Nous ne possédons pour l'instant aucune valeur précise de l'énergie d'activation de ces différents pics pour le titane. Si on compare les spectres obtenus après écrouissage de $10 \%$ sur les deux nuances de titane, on constate que les intensités de relaxation sont sensiblement les mêmes et que la différence la plus caractéristique réside dans la position du maximum : $-6{ }^{\circ} \mathrm{C}$ pour $\mathrm{Ti} 99,97$ et $+30^{\circ} \mathrm{C}$ pour $\mathrm{Ti} 5 \mathrm{~N}$.

En conclusion, les pics «basses températures", $-45^{\circ} \mathrm{C}$ pour $\mathrm{Zr}$ et $-60^{\circ} \mathrm{C}$ pour le titane (fréquence 20 et $16,5 \mathrm{kHz}$ ), dont la « température » ne paraît pas affectée par la pureté et que l'on observe dans tous les cas soit seuls, soit sous forme d'un maximum relatif, seraient consécutifs à un mécanisme propre aux dislocations (kinks). Par contre les pics « hautes températures », très sensibles à la fois à la pureté et à l'écrouissage suggèrent une interaction dislocations-impuretés; on doit noter également que les recuits isochrones entraînent d'abord la décroissance des pics « hautes températures».

La difficulté d'analyse de ces spectres réside précisément dans le recouvrement d'effets qui paraissent correspondre à des mécanismes différents, ce qui entraîne en particulier une grande incertitude sur les énergies d'activation.

\section{Bibliographie}

[1] Boch (P.), Petit .(J.), Gasc (C.), De Fouquet (J.), C. R. Acad. Sci., 266, 605-607.

[2] Bordoni (P. G.), Ric. Sci., 1949, 19, 851 ; NibletT (DH.), Physical Acoustics, Academic Press 1966, III, Chap. 3.

[3] Boch (P.), Mallet (J. M.), De Fouquet (J.), Mem. Sc. Rev. Met., 1967, 64, 507.
[4] Seeger (A.) and ShILler (P.), Physical Acoustic Academic Press, 1965, III, Chap. 8.

[5] Hasiguti, Igata (R. R.), Kamoshita (G. N.), Acta Met., 1962, 10, 442 .

[6] Povolo (F.), Bisogni (E. A.), J. of Nucl. Mat., 1969, $29,82$.

[7] Tsuı (R. T. C.), SACK (A. S.), Acta Met., 1967, 15, 1715 . 